\title{
Endophytic fungal communities associated with field-grown soybean roots and seeds in the Huang-Huai region of China
}

\author{
Hongjun Yang ${ }^{1,2}$, Wenwu Ye Corresp., ${ }^{1,2}$ ， Jiaxin Ma ${ }^{1,2}$ ， Dandan Zeng ${ }^{1,2}$ ，Zhenyang Rong ${ }^{1,2}$, Miao Xu ${ }^{1,2}$, \\ Yuanchao Wang ${ }^{1,2}$, Xiaobo Zheng ${ }^{\text {Corresp. } 1,2}$ \\ 1 Department of Plant Pathology, Nanjing Agricultural University, Nanjing, Jiangsu Province, China \\ 2 The Key Laboratory of Integrated Management of Crop Diseases and Pests (Ministry of Education), Nanjing, Jiangsu Province, China \\ Corresponding Authors: Wenwu Ye, Xiaobo Zheng \\ Email address: yeww@njau.edu.cn, xbzheng@njau.edu.cn
}

Plants depend on beneficial interactions between roots and fungal endophytes for growth, disease suppression, and stress tolerance. In this study, we characterized the endophytic fungal communities associated with the roots and corresponding seeds of soybeans grown in the Huang-Huai region of China. For the roots, we identified 105 and 50 genera by culture-independent and culture-dependent methods, respectively, and isolated 136 fungal strains (20 genera) from the culture-dependent samples. Compared with the 52 soybean endophytic fungal genera reported in other countries, 28 of the genera we found were reported, and 90 were newly discovered. Even though Fusarium was the most abundant genus of fungal endophyte in every sample, soybean root samples from three cities exhibited diverse endophytic fungal communities, and the results between samples of roots and seeds were also significantly different. Together, we identified the major endophytic fungal genera in soybean roots and seeds, and revealed that the diversity of soybean endophytic fungal communities was influenced by geographical effects and tissues. The results will facilitate a better understanding of soybean-endophytic fungi interaction systems and will assist in the screening and utilization of beneficial microorganisms to promote healthy of plants such as soybean. 
1 Endophytic fungal communities associated with field-grown soybean

2 roots and seeds in the Huang-Huai region of China

3 Hongjun Yang ${ }^{1,2}$, Wenwu $\mathrm{Ye}^{1,2^{*}}$, Jiaxin $\mathrm{Ma}^{1,2}$, Dandan Zeng ${ }^{1,2}$, Zhenyang Rong ${ }^{1,2}$, Miao $\mathrm{Xu}^{1,2}$,

4 Yuanchao Wang ${ }^{1,2}$, and Xiaobo Zheng ${ }^{1,2^{*}}$

5

$6{ }^{1}$ Department of Plant Pathology, Nanjing Agricultural University, Nanjing, Jiangsu 210095,

7 China; ${ }^{2}$ The Key Laboratory of Integrated Management of Crop Diseases and Pests (Ministry of

8 Education), Nanjing, Jiangsu 210095, China

9 *E-mail: yeww@njau.edu.cn, xbzheng@njau.edu.cn

10

11 SHORT TITLE:

12 Endophytic fungi communities of soybeans 


\section{ABSTRACT}

16 Plants depend on beneficial interactions between roots and fungal endophytes for growth, disease

17 suppression, and stress tolerance. In this study, we characterized the endophytic fungal communities associated with the roots and corresponding seeds of soybeans grown in the HuangHuai region of China. For the roots, we identified 105 and 50 genera by culture-independent and culture-dependent methods, respectively, and isolated 136 fungal strains (20 genera) from the culture-dependent samples. Compared with the 52 soybean endophytic fungal genera reported in other countries, 28 of the genera we found were reported, and 90 were newly discovered. Even though Fusarium was the most abundant genus of fungal endophyte in every sample, soybean root samples from three cities exhibited diverse endophytic fungal communities, and the results

25 between samples of roots and seeds were also significantly different. Together, we identified the major endophytic fungal genera in soybean roots and seeds, and revealed that the diversity of soybean endophytic fungal communities was influenced by geographical effects and tissues. The results will facilitate a better understanding of soybean-endophytic fungi interaction systems and will assist in the screening and utilization of beneficial microorganisms to promote healthy of plants such as soybean. 


\section{INTRODUCTION}

Fungal endophytes differ from pathogens, which lead to disease and reduce the fitness of their host plants. Fungal endophytes inhabit the asymptomatic aboveground and underground tissues of their hosts and are found in all species and in all divisions of land plants (Zimmerman \& Vitousek 2012). Endophytes often form mutualistic interactions with their host, with the relationship benefitting both partners (Deshmukh et al. 2006). Fungal endophytes and land plants have interacted for over 400 million years (Krings et al. 2007). Since Vogl isolated and cultured symptomless endophytes from seeds of Lolium temulentum (Vogl 1989), fungal endophytes have been studied in many systems, including the diversity and function of clavicipitaceous and nonclavicipitaceous fungal endophytes that infect grasses, nonvascular plants, ferns and allies, conifers, and angiosperms (Clay 1989; Rodriguez et al. 2009). In addition, the relationships between endophytic fungi and medicinal plants have also been reported (Jia et al. 2016).

Endophytes have established long-lasting interactions with different hosts, involving growth stimulation, alleviation of salt stress, and induction of local and systemic resistance to pathogens (Lahrmann et al. 2013). For example, the endophytic fungus, Phomopsis sp., produces piperine, indole-3-acetic acid, and gibberellic acid to promote plant growth (Chithra S et al. 2017), endophytic fungi isolated from the roots of Sophora tonkinensis Gapnep inhibit three fungal phytopathogens of Panax notoginseng (Yao et al. 2017), and endophytic Penicillium strains produce gibberellins to overcome salt stress (Leitao \& Enguita 2016). In addition, the biosynthetic products of the endophytic fungus Nigrospora oryzae, isolated from Combretum dolichopetalum leaves, possess strong antidiabetic activity (Uzor et al. 2017). Endophytic fungi are therefore a source of novel compounds promoting organic farming.

54 In comparison with other crops, studies on endophytes in soybean plants are still limited, 
55 especially those grown in China, a country with a long history of soybean growing. In Brazil, 12

56

57 genera of fungal endophytes were isolated from the leaves and stems of soybeans. There was no difference in the number of fungi isolated from the leaves and stems; however, the number of endophytes decreased as the plants aged, and more fungi were found in tissues near the soil (Pimentel et al. 2006). In Argentina, 11 genera of fungal endophytes were identified in soybean leaves, stems, and roots. Fungal endophytic colonization in soybean plants was influenced by tissue type, with a greater number of endophytes isolated from stems than from leaves and roots (Russo et al. 2016). In the U.S., endophytes in the stems of soybean plants were isolated using culture-dependent $(\mathrm{CD})$ and culture-independent $(\mathrm{CI})$ colony isolation methods, with 12 and 6 genera identified, respectively (Impullitti \& Malvick 2013). Many of the fungal endophytes identified in the above studies are known to be soybean pathogens, e.g., Fusarium and Alternaria; however, they were not able to cause symptoms of disease. It remains to be determined whether those endophytes are latent pathogens or non-pathogenic forms that benefit the host.

For the characterization of endophytic fungal communities, traditional methods of species identification such as morphological observations and sequencing of PCR products to identify molecular marker(s) have been routinely used in previous studies. However, several molecular technologies, e.g., terminal-restriction fragment length polymorphism [T-RFLP], denaturing gradient gel electrophoresis [DGGE], and metagenomics, are now being used to better characterize endophytes and their roles in plant ecophysiology (Rodriguez et al. 2009). For example, DGGE was used to characterize the diversity and phylogenetic relationships of endophytic fungi in Bletilla ochracea (Tao et al. 2008), T-RFLP was used to analyze the structure of ectophytic potato-associated bacterial communities and antagonistic behaviors toward plant pathogenic fungi (Berg et al. 2005), and a metagenomics approach was used to 
78 analyze an endophytic bacterial community in the roots of rice (Sessitsch et al. 2012).

79 In the present study, we characterized the endophytic fungal communities associated with

80 field-grown soybean roots and corresponding seeds in the Huang-Huai region of China, which is

81 one of the most important soybean producing areas. We used both CD and CI methods for

82 colony isolation and sequencing, including PCR sequencing and internal transcribed spacer (ITS)

83 high-throughput sequencing to identify species. The results from different methods, different

84 countries and cities in China, and different tissues (roots and seeds) were compared to evaluate

85 the diversity of endophytic fungal communities associated with soybeans. 
87

88

89

90

91

92

93

94

95

\section{MATERIALS AND METHODS}

\section{Field trials and sampling}

A total of six fields in three cities of the Huang-Huai region of China, Jining of Shandong Province $\left(35^{\circ} 27^{\prime} \mathrm{N}, 116^{\circ} 35^{\prime} \mathrm{E}\right)$, Xuzhou of Jiangsu Province $\left(34^{\circ} 17^{\prime} \mathrm{N}, 117^{\circ} 17^{\prime} \mathrm{E}\right)$, and Suzhou of Anhui Province $\left(33^{\circ} 38^{\prime} \mathrm{N}, 117^{\circ} 05^{\prime} \mathrm{E}\right)$, were selected for this study; the corresponding samples were named JN1 and JN2, XZ1 and XZ2, and SZ1 and SZ2, respectively. The two fields in each city were adjacent, and each field was $30 \mathrm{~m} \times 8 \mathrm{~m}$ in size. The fields had been under a similar agronomic management and fertilization regime for 3 years. The crop planting pattern was a soybean-wheat rotation, and the soybean cultivar was ZhongHuang 13, which is widely grown in Huang-Huai region of China.

The seeds to be grown were harvested from the previous year; those harvested in 2015 and sown in 2016 were sampled for assays, with a suffix "a" at the end of the sample name. The seeds were stored at $4^{\circ} \mathrm{C}$ until analyzed. Healthy adult soybean plants were sampled during July and September of 2016. Each sampling was comprised of 15 soybean plants collected in the shape of a W from 15 sites in each field. The time points for sampling were 30, 60, and 90 days after seed sowing, which corresponded to the flowering, pod formation, and maturation stages of the soybean growth period, with suffixes of "b," "c," and "d" at the end of the sample names, respectively. The loosely adhering soil on the roots was removed by shaking, immediately placed into sterile plastic bags in an ice box, and then transported to the laboratory for analyses.

\section{Pretreatment of the samples}

After a washing step to remove soil residue and dust, the roots of each plant were cut into segments of 5-7 cm, and $2 \mathrm{~g}$ of each root segment was analyzed. Fifteen plants collected from 
110 the same field were pooled, and $10 \mathrm{~g}$ of the pooled root segment sample were selected randomly.

111 The $10 \mathrm{~g}$ root segments were supplemented with $200 \mathrm{~mL}$ distilled water and three drops of

112 Tween 20 at $25^{\circ}$, then shaken at $220 \mathrm{rpm}$ for $20 \mathrm{~min}$. We also randomly selected $10 \mathrm{~g}$ of the seed

113 sample for analyses. Surface sterilization was performed following previous protocols (Impullitti

114 \& Malvick 2013; Potshangbam et al. 2017) with some modifications; each root and seed was

115 successively washed with sterile water for $20 \mathrm{~s}$, immersed in $70 \%$ ethanol for $5 \mathrm{~s}$, soaked in

$1160.525 \%$ sodium hypochlorite for $2 \mathrm{~min}$, washed with sterile distilled water again three or four

117 times, then dried under sterile conditions. Each sample was divided into two parts, which were

118 used for the CD and CI methods, as described below.

119

120 The CD method

121 The CD method was used to obtain pure fungal colonies for further identification of individual

122 species and to obtain mixed fungal colonies for ITS sequencing. The root segments were further 123 cut into shorter segments $(0.25 \mathrm{~cm})$, and every five randomly-selected segment was placed on a 124 plate containing potato dextrose agar (PDA), malt extract agar, synthetic potato medium, and 125 czapek dox medium. All culture media were also amended with ampicillin (50 mg/L) and

126 rifampicin $(50 \mathrm{mg} / \mathrm{L})$ to inhibit bacterial growth. There were a total of 360 pieces $(5$ segments per plate $\times 3$ plates $\times 4$ culture media $\times 6$ fields), which were maintained at $25^{\circ} \mathrm{C}$.

To identify the species of pure fungal colonies, after 3-5 days, when mycelia emerged from the root tissues, small pieces of medium together with mycelia from the margins of growing cultures were transferred to a new PDA plate. These pure fungal cultures were preliminarily grouped into morphotaxa, based on mycelium type, colony color, and growth rate. After 7 days of growth, mycelia DNA was extracted using the DNAsecure Plant Kit (Tiangen, Beijing, China) 
133 according to the manufacturer's instructions. Nuclear rDNA regions, including ITS1, 5.8S, and

134 ITS2, were amplified by PCR in a $30 \mu \mathrm{L}$ reaction containing $15 \mu \mathrm{L} 2 \times$ EasyTaq PCR SuperMix

135 (+dye) (TransGen, Beijing, China), $1 \mu \mathrm{L}$ each of the primers ITS1-F and ITS4 (Table 1), $1 \mu \mathrm{L}$

136 template DNA solution, and $12 \mu \mathrm{L}$ sterile water, using previously described reaction parameters

137 (Liang et al. 2014). For Fusarium species, we also used primers EF-1 and EF-2 to amplify the

138 translation elongation factor (EF)-1 $\alpha$ region for confirmation (O'Donnell et al. 2010) (Table 1).

139 The PCR products were sequenced, and the sequences were compared with the NCBI database

140 (www.ncbi.nlm.nih.gov; using the BLASTN program) for identification of species.

141 For ITS sequencing of mixed fungal colonies, after 3 weeks of fungal growth in root

142 segments, the mycelia on the surface of the culture medium were scraped, and those from the

143 same type of culture medium were pooled for DNA extraction. Equal volume of DNA from

144 mycelia grown on four different kinds of culture medium were mixed into one sample for ITS

145 sequencing as described below.

146

147 The CI method

148 Ten grams of the root or seed samples were frozen and ground in liquid nitrogen using a mortar

149 and pestle. Approximately $400 \mathrm{mg}$ each sample were used to extract genomic DNA according to 150 the method described above.

151

152 MiSeq sequencing and data analyses

153 ITS sequencing for identification of fungi was performed following a previous protocol (Kozich 154 et al. 2013). The ITS1 region of the fungal ITS was amplified using the ITS1-F and ITS2 primers 155 (Table 1). After successful amplification, the PCR products were purified using AmpureXP 
156 beads (Agencourt Bioscience, Beverly, MA, USA) to remove nonspecific products. The libraries

157 were evaluated as follows: (1) the average molecule length was determined using the Agilent 1582100 bioanalyzer and Agilent DNA 1000 kit (Agilent Technologies, Santa Clara, CA, USA), and 159 (2) quantitative real-time PCR (qRT-PCR) using EVAGreen ${ }^{\mathrm{TM}}$ (Jena Bioscience, Jena, Germany) 160 was used to quantitate the libraries. The final libraries were sequenced using the MiSeq system 161 and the MiSeq reagent kit (Illumina, San Diego, CA, USA) using PE250 (Illumina).

162 The raw sequence reads were first filtered according to the default parameters. The 163 overlapping clean reads were assembled into consensus sequences (namely tags) using the Fast

164

165 166
Length Adjustment of Short reads, version 1.2.11 (ccb.jhu.edu/software/FLASH) program. The tags were further clustered into operational taxonomic units (OTUs) with a sequence identity of 97\% as the threshold using UPARSE (www.drive5.com/uparse), and chimeras were filtered out using UCHIME, version 4.2.40 (drive5.com/uchime). The OTUs were taxonomically classified using Ribosomal Database Project Classifier, version 2.2 (rdp.cme.msu.edu), referencing the UNITE ITS database (unite.ut.ee) using 0.6 confidence values as the cutoff.

\section{Statistical analysis}

We performed $\alpha$-diversity and principal coordinate analyses using $\mathrm{R}$ package (version 3.1.1; www.r-project.org). Data from the different bioassays were compared by one-way analysis of variance using SPSS Statistics 20 software for Windows (SPSS, Chicago, IL, USA). The Pearson and Spearman correlations for data from selected taxonomic groups (phyla or genera) were also calculated using this software. 
178

179

180

181

182

183

184

185

186

187

188

189

190

191

192

193

194

195

196

197

198

199

200

\section{RESULTS}

\section{Endophytic fungal communities in the collected healthy soybean root samples}

We analyzed endophytic fungal communities by high-throughput ITS sequencing of 18 CI root samples (CI-seq), e.g., the 6 samples from Jining were designated as JN1b, JN1c, JN1d, JN2b, JN2c, and JN2d. The resulting high-quality reads with $>97 \%$ sequence identity were clustered into 385 OTUs, ranging from 37 to 116 OTUs per sample. Annotation of these OTUs resulted in identification of 105 fungal genera (Table S1). We also analyzed 18 CD root samples by highthroughput ITS sequencing (CD-seq) and identified a total of 50 fungal genera (Table S1). In addition, 136 fungal strains belonging to 20 genera were isolated from the CD root samples (CDiso; Table S2). Thus according to the number of genera, the throughputs of the three methods ranked as CI-seq $>$ CD-seq $>$ CD-iso.

Among all identified 142 genera, 98 were only identified using the high-throughput ITS sequencing method (CI-seq and CD-seq) rather than CD-iso. The OTU abundances of the 98 genera were $14.6 \%$ by CI-seq and $1.8 \%$ by CD-seq. Twelve genera were identified by the CD method only (CD-seq and CD-iso) rather than CI-seq. The OTU abundances were $0.2 \%$ by CDseq and $15.4 \%$ by CD-iso.

Twelve genera were identified by all three methods (Figure 1A). The OTU abundances of these 12 genera were over $80 \%(85.4 \%, 98.2 \%$, and $83.8 \%)$ of all genera identified by CI-seq, CD-seq, and CD-iso, respectively (Table S1). Among the 12 genera, Fusarium exhibited the highest abundance using all three methods; the OTU abundances of Fusarium were over half using CI-seq (62.2\%) and CD-seq (67.8\%), and 39.7\% using CD-iso (Figure 1B; Table S1). The abundances of the other genera were much lower than Fusarium and varied depending on the method of analysis. For example, the second most abundant genera using CI-seq, CD-seq, and 
201 CD-iso were Rhizoctonia (11.7\%), Clonostachys (14.3\%), and Trichoderma (16.9\%), 202 respectively (Figure 1B; Table S1).

\section{Geographical effects on soybean root endophytic fungal communities}

204 We further analyzed the CI-seq results of samples from three different cities to determine if

205

206

207

208

209

210

211

212

213

214

215

216

217

218

219

220

221

222

223 geographical location affected the root endophytic fungal communities. We identified 77, 80, and 75 fungal genera from Jining, Suzhou, and Xuzhou, respectively, and 25, 28, and 23 genera, respectively, were not identified in at least one other city (Figure 2A; Table S3). However, the OTU abundances of these non-core genera were only $0.4 \%, 0.6 \%$, and $0.3 \%$ of all genera in the corresponding cities, respectively (Table S3).

Principal coordinate analyses of weighted distances were performed to investigate patterns of separation between microbial communities. The samples from Xuzhou were separated from those of Jining and Suzhou along the first principal coordinate, PCo1, and the samples of Jining were separated from the others along the principal coordinate, $\mathrm{PCo} 2$, indicating that endophytic fungal communities varied among the different cultivation sites (Figure 2B). Analyses of withinsample diversity ( $\alpha$-diversity or Shannon' diversity) showed that endophytic fungal communities of Jining had the highest diversity, in contrast to samples from Suzhou that were significantly different (Figure 2C).

The geographical effects on the soybean root endophytic fungal communities were present in the dominant core genera. For example, there were 52 fungal genera shared in all three cities, and the top five most frequent genera were Fusarium, Rhizoctonia, Phoma, Clonostachys, and Macrophomina. Among the results from three cities, Suzhou had the highest OTU abundance in Fusarium and Macrophomina, Xuzhou had the highest OTU abundance in Rhizoctonia, Clonostachys, and Calonectria, and the lowest OTU abundance in Phoma (Figure 2D; Table 
224 S3).

225

\section{Significant differences in endophytic fungal communities between roots and seeds}

227 The endophytic fungal species detected in plants could be influenced by many factors, such as

228 the type of tissue. We further compared the CI-seq results of endophytic fungal communities

229 between the sampled roots and corresponding seeds for soybean plant growth. There were 38

230 common fungal genera between the root and seed samples, with 67 genera unique in roots, while

231 only 11 genera were unique in seeds (Figure 3A; Table S4).Using principal coordinate analyses

232 analyses, the root and seed samples were clearly distinct along the the first principal coordinate

233 (PCo1; Figure 3B).

234 The most abundant annotated genera in roots were Fusarium, Rhizoctonia, Phoma, 235 Clonostachys, Mortierella, and Calonectria, and the OTU abundances of these genera were all 236 higher than those in seeds. Genera such as Macrophomina and Ilyonectria were identified in 237 roots only (Figure 3C; Table S4). In contrast, Cladosporium, Alternaria, Engyodontium, 238 Guehomyces, Nigrospora, and Aspergillus were more abundant in seeds than in roots (Figure 3C; 239 Table S4). 


\section{DISCUSSION}

242 To the best of our knowledge, this is the first report of fungal endophytic diversity in field-grown

243 soybean roots in the Huang-Huai region of China. Three methods of analyses were used to obtain

244 a complete qualitative picture of endophytic fungal communities. Compared with the CD-iso

245 method, the high-throughput sequencing-based CD-seq and CI-seq methods showed significant

246 advantages in terms of sensitivity. The CI-seq method allows detection and identification of all

247 fungi including those that are cryptic, sterile, and unculturable, and potentially detects a more

248 diverse endophyte population than CD-iso methods (Impullitti \& Malvick 2013). However, the

249 CD-seq and CI-seq methods amplified shorter DNA fragment and their identifications were

250 subsequently compromised to only genus-level. Compared with the CI-seq method, CD-iso

251 method recovered the lowest quantity of fungal population, but its identification had the best

252 quality. Most fungal isolates were identified to species-level (Table S2). In addition, CD-iso

253 method could obtain fungal culture, and provides fundamental materials for studying the

254 biological and ecological roles in subsequent research. Interestingly, some fungi were only found

255 by isolation, and the reason has not yet been explained.

256 Of 52 previously reported endophytic fungal genera, 28 (53.8\%) were identified in our study,

257

258

259

260

261

262

263 and $25(89.2 \% ; 25 / 28)$ of these genera were identified using CI-seq (Figure 1A; Table S1). The remaining 24 of the 52 reported genera were not identified by any method in our study (Table S1), possibly because the analyzed samples were collected from different tissues such as leaves or stems, and the soybean plants were different cultivars and grown under different environments. There were 90 genera newly identified in our study. Genera such as Rhizoctonia and Clonostachys, which showed high OTU abundances by high-throughput ITS sequencing (CI-seq and CD-seq) but low by CD-iso, have not been reported previously (Figure 1B; Table S1), 
264 possibly because previous studies used CD-iso- or CD-iso-like methods, rather than high265 throughput methods. Fusarium was also reported as endophytic fungi in soybean roots and 266 leaves, further revealing that it is a dominant fungi species in soybean endophytic fungal 267 community.

268 Many soybean pathogens colonize roots, and the caused root diseases lead to extensive losses 269 in soybean production worldwide. Over the long period of coexistence and evolution, different 270 relationships have been established between endophytic fungi and their host plants through 271 specific fungus-host interactions identified as a continuum of mutualism, antagonism, and 272 neutralism (Jia et al. 2016). It is therefore valuable not only to identify the pathogens in disease 273 soybean roots but also to systematically identify the microbial communities in healthy roots. We 274 found many fungal genera/species in healthy soybean roots that are well-known pathogen species 275 of soybean and/or other plants such as wheat. For example, Fusarium, Macrophomina, 276 Rhizoctonia, and Alternaria cause root rot or foliar and stem blight in soybeans (Lu et al. 2015a; 277 Lu et al. 2015b; Ramos et al. 2016; Yamamoto et al. 2017). Our preliminary pathogenicity assays for some of these isolates showed that many of them may be truly asymptomatic in soybean seedlings, while some were able to cause disease symptoms, indicating that they may be latent pathogens that cause disease under stress or at a later time during plant growth. Other identified endophytic fungal genera, such as Ceratobasidium and Phoma, have rarely been associated with soybean diseases (Impullitti \& Malvick 2013). However, these genera have been reported to be major pathogens in other plants such as wheat (Kriuchkova 2013; Perello \& Moreno 2005). Among the endophytic fungi isolated from soybean, a high dominance was observed in the roots of Fusarium, and Fusarium has been isolated from various parts of the 286 soybean plant (Pimentel et al. 2006; Russo et al. 2016; Impullitti \& Malvick 2013), which can be 
287 worrisome because it undetermined whether these isolates are non-pathogenic and beneficial to 288 the plant or if they are latent pathogens. Overall, the significance of endophytism of endophytes 289 in soybeans is still largely unknown and merits further investigation. as the type of tissue sampled and the location in which they are grown. Greater numbers of endophytes were isolated from stem tissues than from leaves and root tissues in both soybeans and corn (Russo et al. 2016). In the medicinal plant Kadsura angustifoli, the fungal species diversity differed significantly between stems and roots, with roots containing a greater diversity than stems (Huang et al. 2015). There were significant differences in the endophytic communities among different regions of the Great Lakes Basin (Clay et al. 2016). Another study reported significant site and location variations in fungal endophytic communities (Zimmerman endophytic fungal communities. The numbers of fungal genera were twofold greater in roots than in seeds, while the number of root-specific fungal genera were six-fold greater than that of seed-specific fungal genera, suggesting that the field environment, such as the soil conditions, affected the endophytic fungal communities of soybean plants after the seeds have been sown. The root specific fungi might initially be present in the soil, followed by colonization of the roots and the entire plant after seed germination. Because of different soil properties among the three cities (JN, a sandy loam soil; XZ, a yellow loam sand soil; SZ, a mortar black soil), the endophytic fungal communities were also diverse. Soil type and growth conditions also shape 
308 and soils is the main driver of fungal alpha diversity, the plant growing condition is the main

309 factor structuring root-associated fungal communities, i.e., determining the taxa present and their

310 abundances (Almario, et al., 2017). Thus the specific impact of each environment factor still

311 needs to be identified in future studies.

312 


\section{CONCLUSIONS}

314 In this study, we characterized the endophytic fungal communities associated with the roots and 315 corresponding seeds of soybeans grown in the Huang-Huai region of China. We identified the 316 major endophytic fungal genera of soybeans and revealed that the diversity of soybean 317 endophytic fungal communities was influenced by geographical effects and tissues. The results 318 will facilitate a better understanding of soybean-endophytic fungi interaction systems and will 319 assist in the screening and utilization of beneficial microorganisms to promote healthy of plants 320 such as soybean. 


\section{REFERENCES}

324

325

326

327

328

329

330

331

332

333

334

335

336

337

338

339

340

341

342

343

344

345

346

347

348

349

350

351

352

353

354

355

356

357

358

359

360

361

362

363
Almario J, Jeena G, Wunder J, Langen G, Zuccaro A, Coupland G, and Bucher M. 2017.Root-associated fungal microbiota of nonmycorrhizal Arabis alpina and its contribution to plant phosphorus nutrition. Proc Natl Acad Sci U S A 114: E9403-E9412.

Berg G, Krechel A, Ditz M, Sikora RA, Ulrich A, and Hallmann J. 2005. Endophytic and ectophytic potatoassociated bacterial communities differ in structure and antagonistic function against plant pathogenic fungi. FEMS Microbiol Ecol 51:215-229. 10.1016/j.femsec.2004.08.006

Chithra S, Jasim B, Jyothis Mathew, and Radhakrishnan EK. 2017. Endophytic Phomopsis sp. colonization in Oryza sativa was found to result in plant growth promotion and piperine production. Physiol Plant 160:437-446. $10.1111 / \mathrm{ppl} .12556$

Clay K. 1989. Clavicipitaceous endophytes of grasses: their potential as biocontrol agents. Mycological Research 92:1-12.

Clay K, Shearin ZRC, Bourke KA, Bickford WA, and Kowalski KP. 2016. Diversity of fungal endophytes in nonnative Phragmites australis in the Great Lakes. Biological Invasions 18:2703-2716.

Deshmukh S, Huckelhoven R, Schafer P, Imani J, Sharma M, Weiss M, Waller F, and Kogel KH. 2006. The root endophytic fungus Piriformospora indica requires host cell death for proliferation during mutualistic symbiosis with barley. Proc Natl Acad Sci U S A 103:18450-18457. 10.1073/pnas.0605697103

Huang Q, An H, Song H, Mao H, Shen W, and Dong J. 2015. Diversity and biotransformative potential of endophytic fungi associated with the medicinal plant Kadsura angustifolia. Res Microbiol 166:45-55. 10.1016/j.resmic.2014.12.004

Impullitti AE, and Malvick DK. 2013. Fungal endophyte diversity in soybean. J Appl Microbiol 114:1500-1506. 10.1111/jam. 12164

Jia M, Chen L, Xin HL, Zheng CJ, Rahman K, Han T, and Qin LP. 2016. A friendly relationship between endophytic fungi and medicinal plants: a systematic review. Front Microbiol 7:906. 10.3389/fmicb.2016.00906

Kozich JJ, Westcott SL, Baxter NT, Highlander SK, and Schloss PD. 2013. Development of a dual-index sequencing strategy and curation pipeline for analyzing amplicon sequence data on the MiSeq Illumina sequencing platform. Appl Environ Microbiol 79:5112-5120. 10.1128/aem.01043-13

Krings M, Taylor TN, Hass H, Kerp H, Dotzler N, and Hermsen EJ. 2007. Fungal endophytes in a 400-million-yrold land plant: infection pathways, spatial distribution, and host responses. New Phytol 174:648-657. 10.1111/j.1469-8137.2007.02008.x

Kriuchkova LO. 2013. Micromycetes associated with wheat diseases in different regions of Ukraine. Mikrobiol Z 75:59-68.

Lahrmann U, Ding Y, Banhara A, Rath M, Hajirezaei MR, Dohlemann S, von Wiren N, Parniske M, and Zuccaro A. 2013. Host-related metabolic cues affect colonization strategies of a root endophyte. Proc Natl Acad Sci USA 110:13965-13970. 10.1073/pnas.1301653110

Leitao AL, and Enguita FJ. 2016. Gibberellins in Penicillium strains: Challenges for endophyte-plant host interactions under salinity stress. Microbiological Research 183:8-18.

Liang J, Sun S, Ji J, Wu H, Meng F, Zhang M, Zheng X, Wu C, and Zhang Z. 2014. Comparison of the rhizosphere bacterial communities of Zigongdongdou soybean and a high-methionine transgenic line of this cultivar. PLoS One 9:e103343. 10.1371/journal.pone.0103343 
364

365

366

367

368

369

370

371

372

373

374

375

376

377

378

379

380

381

382

383

384

385

386

387

388

389

390

391

392

393

394

395

396

397

398

399

400

401

402

403

404

Lu C, Song B, Zhang H, Wang Y, and Zheng X. 2015a. Rapid diagnosis of soybean seedling blight caused by Rhizoctonia solani and soybean charcoal rot caused by Macrophomina phaseolina using LAMP assays. Phytopathology 105:1612-1617. 10.1094/PHYTO-01-15-0023-R

Lu C, Zhang H, Wang Y, and Zheng X. 2015b. Rapid diagnosis of Fusarium root rot in soybean caused by Fusarium equiseti or Fusarium graminearum using loop-mediated isothermal amplification (LAMP) assays. Australasian Plant Pathology 44:437-443. 10.1007/s13313-015-0361-8

O'Donnell K, Sutton DA, Rinaldi MG, Sarver BA, Balajee SA, Schroers HJ, Summerbell RC, Robert VA, Crous PW, Zhang N, Aoki T, Jung K, Park J, Lee YH, Kang S, Park B, and Geiser DM. 2010. Internet-accessible DNA sequence database for identifying fusaria from human and animal infections. J Clin Microbiol 48:3708-3718. 10.1128/jcm.00989-10

Perello AE, and Moreno MV. 2005. First report of Phoma sorghina (Sacc.) Boerema Dorenbosch \& van Kest on wheat leaves (Triticum aestivum L.) in Argentina. Mycopathologia 159:75-78.

Pimentel IC, Glienke-Blanco C, Gabardo J, Stuart RM, and Azevedo JL. 2006. Identification and colonization of endophytic fungi from soybean (Glycine $\max (\mathrm{L}$.) Merril) under different environmental conditions. Brazilian Archives of Biology and Technology 49:705-711.

Potshangbam M, Devi SI, Sahoo D, and Strobel GA. 2017. Functional characterization of endophytic fungal community associated with Oryza sativa L. and Zea mays L. Front Microbiol 8:325. 10.3389/fmicb.2017.00325

Ramos AM, Gally M, Szapiro G, Itzcovich T, Carabajal M, and Levin L. 2016. In vitro growth and cell wall degrading enzyme production by Argentinean isolates of Macrophomina phaseolina, the causative agent of charcoal rot in corn. Rev Argent Microbiol 48:267-273. 10.1016/j.ram.2016.06.002

Rodriguez RJ, White JF, Jr., Arnold AE, and Redman RS. 2009. Fungal endophytes: diversity and functional roles. New Phytol 182:314-330. 10.1111/j.1469-8137.2009.02773.x

Russo ML, Pelizza SA, Cabello MN, Stenglein SA, Vianna MF, and Scorsetti AC. 2016. Endophytic fungi from selected varieties of soybean (Glycine max L. Merr.) and corn (Zea mays L.) grown in an agricultural area of Argentina. Rev Argent Microbiol 48:154-160. 10.1016/j.ram.2015.11.006

Sessitsch A, Hardoim P, Doering J, Weilharter A, Krause A, Woyke T, Mitter B, Hauberg-Lotte L, Friedrich F, Rahalkar M, Hurek T, Sarkar A, Bodrossy L, van Overbeek L, Brar D, van Elsas JD, and Reinhold-Hurek B. 2012. Functional characteristics of an endophyte community colonizing rice roots as revealed by metagenomic analysis. Molecular Plant-Microbe Interactions 25:28-36.

Tao G, Liu ZY, Hyde KD, Liu XZ, and Yu ZN. 2008. Whole rDNA analysis reveals novel and endophytic fungi in Bletilla ochracea (Orchidaceae). Fungal Diversity 33:101-122.

Uzor PF, Osadebe PO, and Nwodo NJ. 2017. Antidiabetic activity of extract and compounds from an endophytic fungus Nigrospora oryzae. Drug Res (Stuttg) 67:308-311. 10.1055/s-0042-122777

Vogl A. 1989. Mehl und die anderen mehl produkte der cerealien und leguminosen. Zeitschrift Nahrungsmittle untersuchung, Hgg Warlenkunde 21:25-29.

Yamamoto R, Nakagawa A, Shimada S, Komatsu S, and Kanematsu S. 2017. Histopathology of red crown rot of soybean. Journal of General Plant Pathology 83:23-32.

Yao YQ, Lan F, Qiao YM, Wei JG, Huang RS, and Li LB. 2017. Endophytic fungi harbored in the root of Sophora tonkinensis Gapnep: Diversity and biocontrol potential against phytopathogens. Microbiologyopen 6.

Zimmerman NB, and Vitousek PM. 2012. Fungal endophyte communities reflect environmental structuring across a

PeerJ reviewing PDF | (2018:02:25939:1:1:CHECK 12 Apr 2018) 


\section{ADDITIONAL INFORMATION}

409 Supplementary Information accompanies this paper.

410 - Table S1. The identified fungal genera using three different methods

411 - Table S2. Endophytic fungal species isolated from healthy soybean roots (CD-iso)

412 - Table S3. Fungal genera identified from different cities

413 - Table S4. Fungal genera identified from different tissues (roots and seeds) 


\section{FIGURE LEGENDS}

416 Figure 1. Root-associated endophytic fungi detected using three different methods. (A) Number

417 of endophytic fungal genera detected using different methods. The numbers of previously 418 reported endophytic fungal genera were also indicated in parentheses. (B) The relative 419 abundances of the indicated endophytic fungal genera. Culture dependent-specific fungal genera 420 were only identified using CD-seq and/or CD-iso, and sequencing-specific fungal genera were 421 only identified using CI-seq and/or CD-seq. Shared fungal genera were identified by all three 422 methods. "*”, fungal genera that have been reported as endophytic fungi.

Figure 2. Root-associated endophytic fungi identified at three locations. (A) The number of endophytic fungal genera detected from three locations. (B) The results of principal coordinate analyses; every sample is labeled with a specific color to designate the location. (C) Withinsample diversity ( $\alpha$-diversity or Shannon's diversity) analyses of root samples. The horizontal bars within boxes represent the medians. The upper and lower edges of the boxes represent the $75^{\text {th }}$ and $25^{\text {th }}$ quartiles, respectively. The upper and lower whiskers extend 1.5 -fold of the interquartile range from the upper and lower edges of the box. (D) The relative abundances (\%) of the indicated endophytic fungal genera from three locations.

433 Figure 3. Endophytic fungi detected in seeds and roots of soybeans. (A) Numbers of endophytic 434 fungal genera detected from root and seed samples. (B) The results of principal coordinate 435 analyses; every sample is labeled with a specific color to designate the tissue. (C) Relative abundances $(\%)$ of the indicated endophytic fungal genera in the root and seed samples. 


\section{Table $\mathbf{1}$ (on next page)}

The primers used in this study 
1 Table 1. The primers used in this study

\begin{tabular}{llll}
\hline Assay & Target gene (region) & Primer & Sequence (5'-3') \\
\hline Regular PCR & ITS1, 5.8S, and ITS2 & ITS1-F & CTTGGTCATTTAGAGGAAGTAA \\
& & ITS4 & TCCTCCGCTTATTGATATGC \\
Regular PCR & EF-1 $\alpha$ & EF-1 & ATGGGTAAGARGACAAGAC \\
& & EF-2 & GGARGTACCAGTSATCATGTT \\
Amplicon sequencing & ITS1 & ITS1-F & CTTGGTCATTTAGAGGAAGTAA \\
& & ITS2 & GCTGCGTTCTTCATCGATGC \\
\hline
\end{tabular}

2 


\section{Figure 1}

Root-associated endophytic fungi detected using three different methods

(A) Number of endophytic fungal genera detected using different methods. The numbers of previously reported endophytic fungal genera were also indicated in parentheses. (B) The relative abundances of the indicated endophytic fungal genera. Culture dependent-specific fungal genera were only identified using CD-seq and/or CD-iso, and sequencing-specific fungal genera were only identified using $\mathrm{Cl}$-seq and/or CD-seq. Shared fungal genera were identified by all three methods. "*", fungal genera that have been reported as endophytic fungi.

A

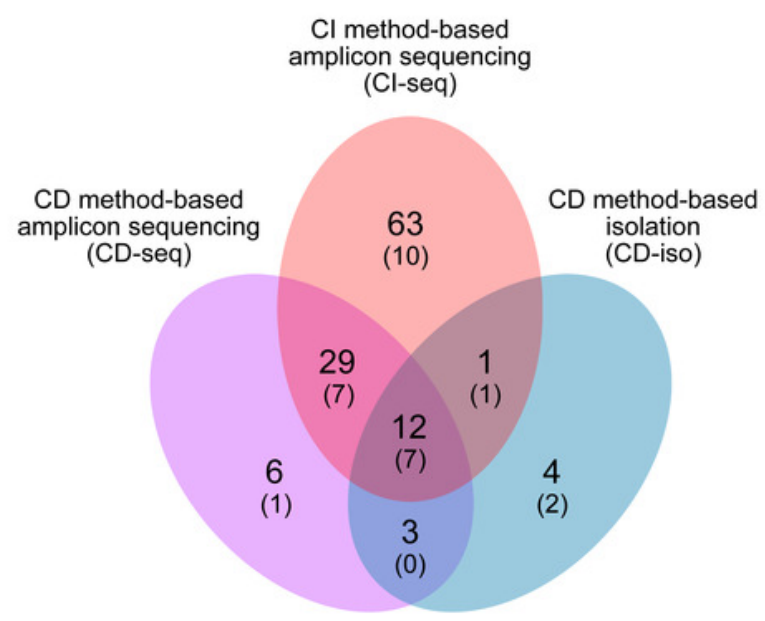

Number of identified fungi genera
B

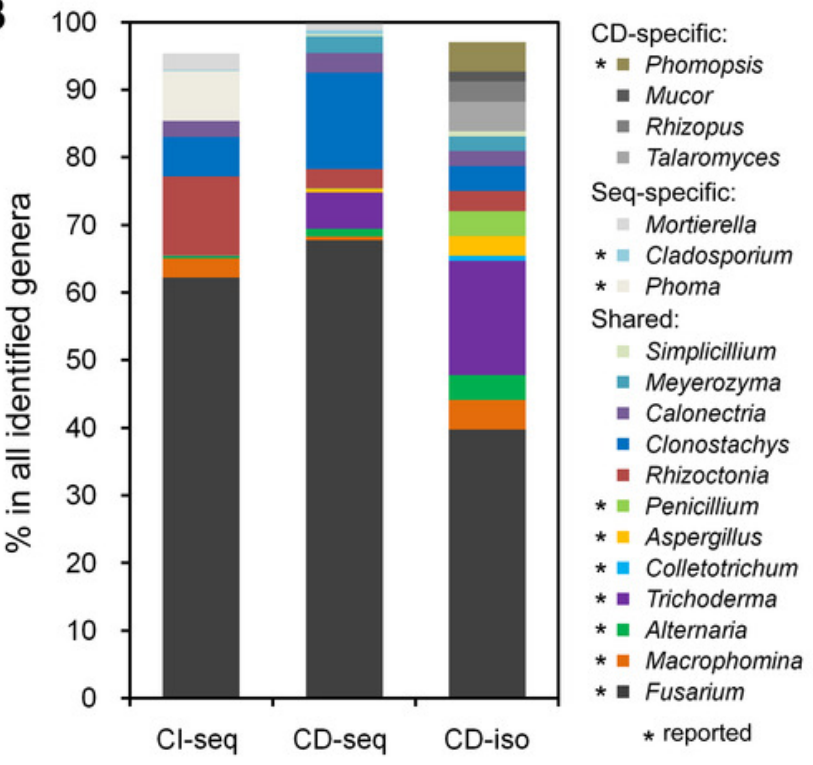




\section{Figure 2}

Root-associated endophytic fungi identified at three locations

(A) The number of endophytic fungal genera detected from three locations. (B) The results of principal coordinate analyses; every sample is labeled with a specific color to designate the location. (C) Within-sample diversity ( $\alpha$-diversity or Shannon's diversity) analyses of root samples. The horizontal bars within boxes represent the medians. The upper and lower edges of the boxes represent the $75^{\text {th }}$ and $25^{\text {th }}$ quartiles, respectively. The upper and lower whiskers extend 1.5 -fold of the interquartile range from the upper and lower edges of the box. (D) The relative abundances (\%) of the indicated endophytic fungal genera from three locations. 
A

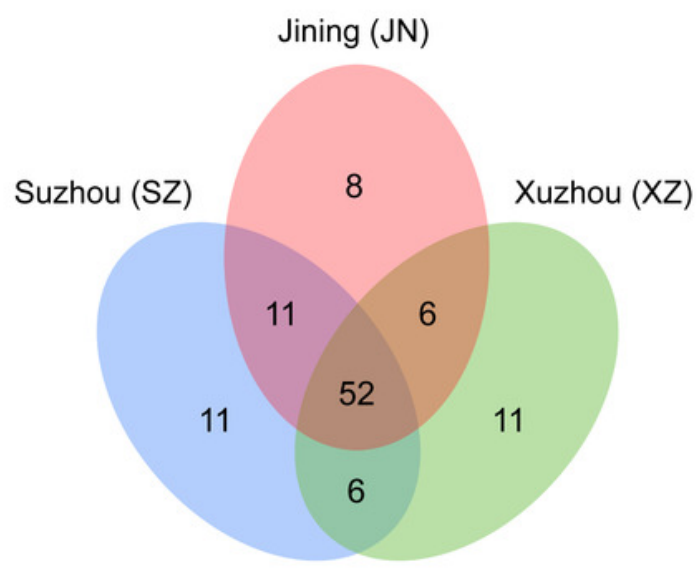

Number of identified fungi genera

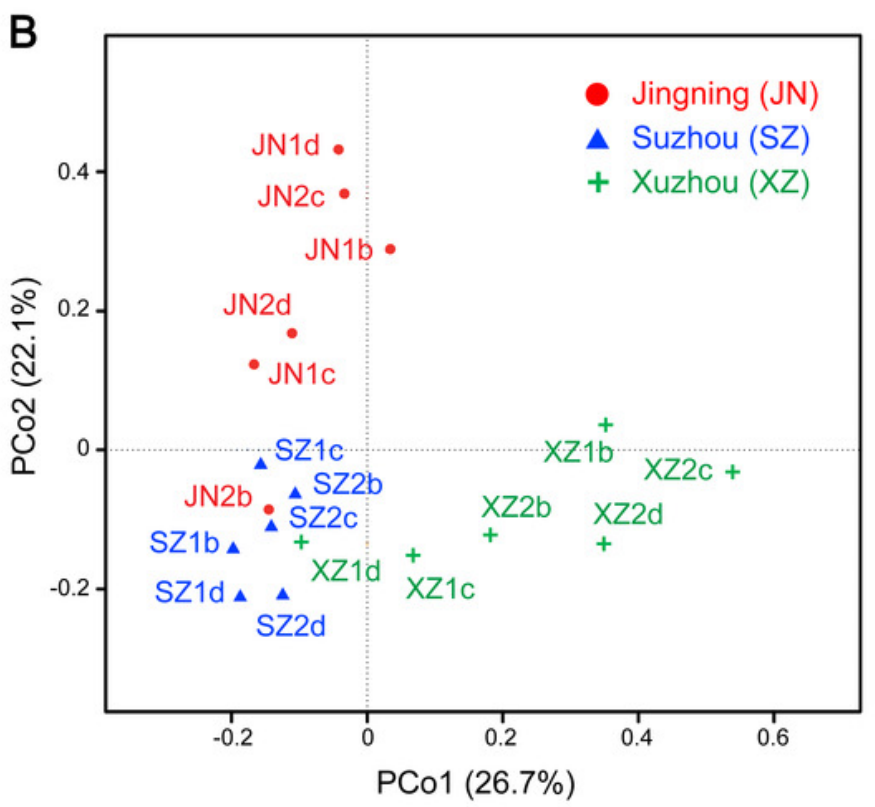

C
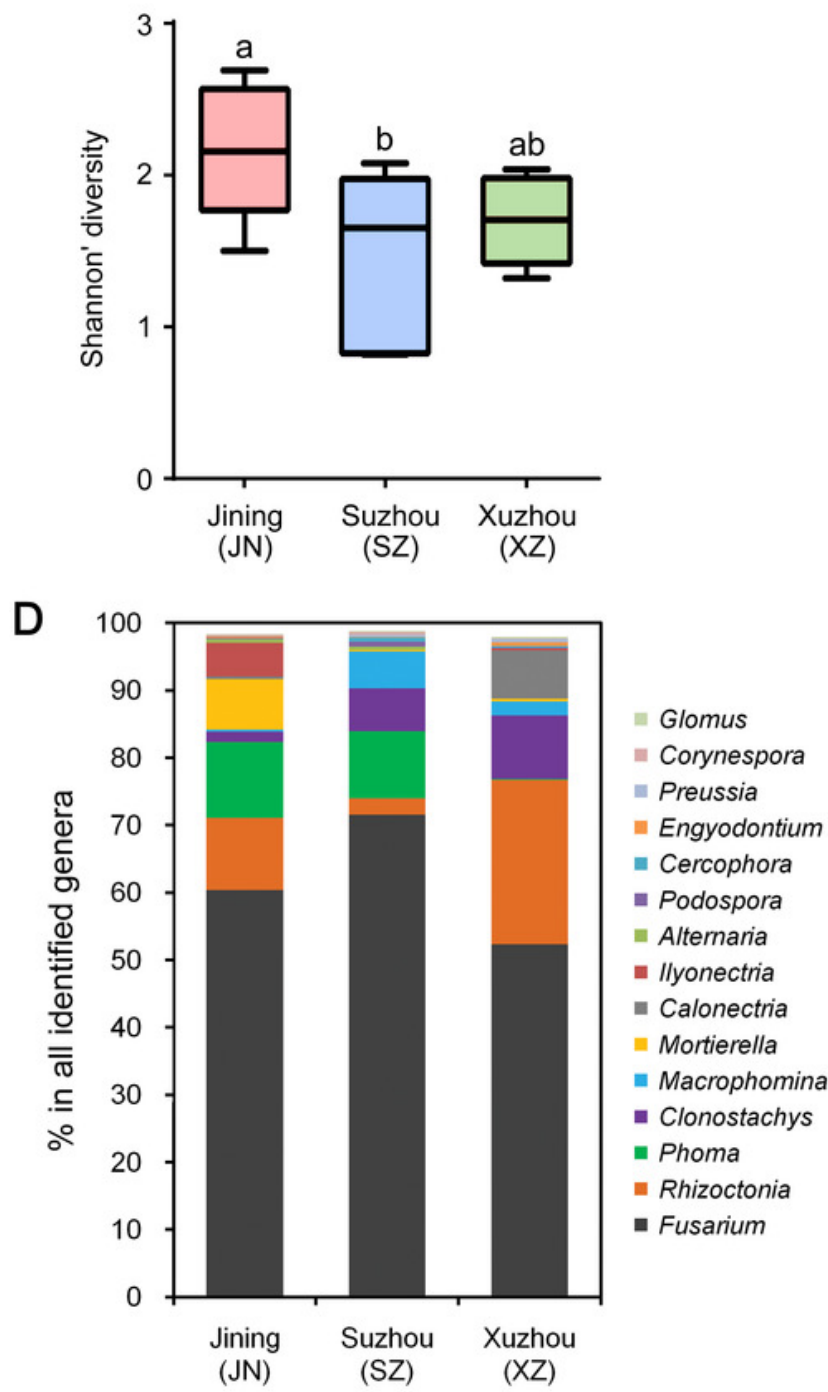
Figure 3

Endophytic fungi detected in seeds and roots of soybeans

(A) Numbers of endophytic fungal genera detected from root and seed samples. (B) The results of principal coordinate analyses; every sample is labeled with a specific color to designate the tissue. (C) Relative abundances (\%) of the indicated endophytic fungal genera in the root and seed samples.

A

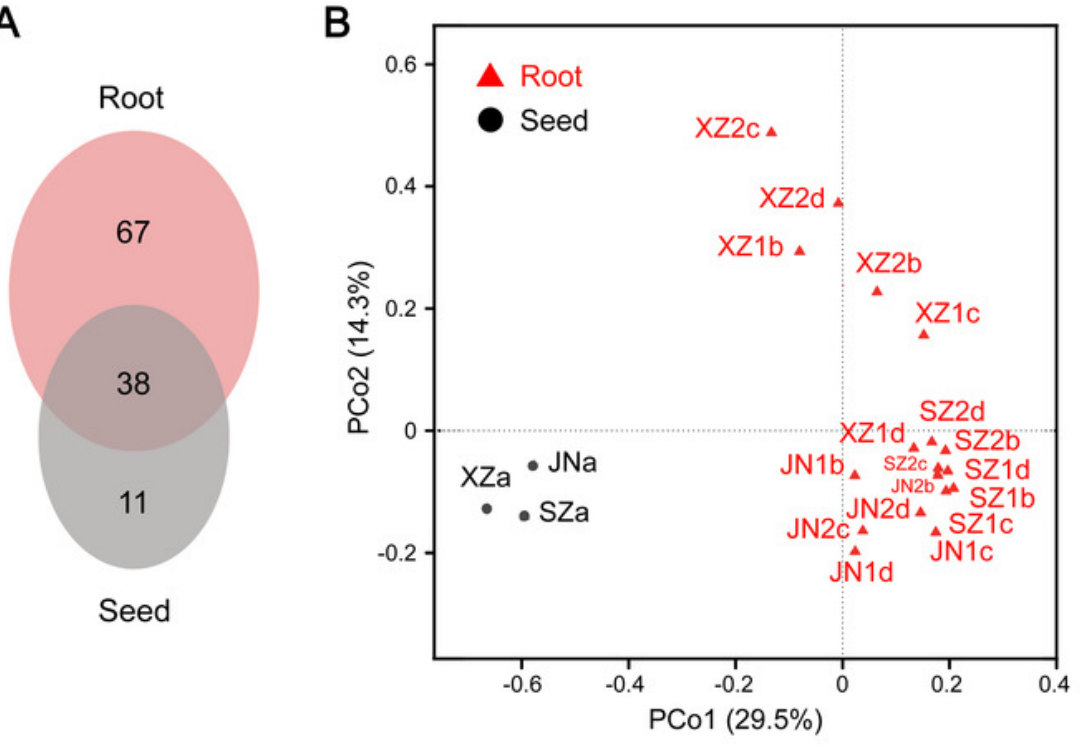

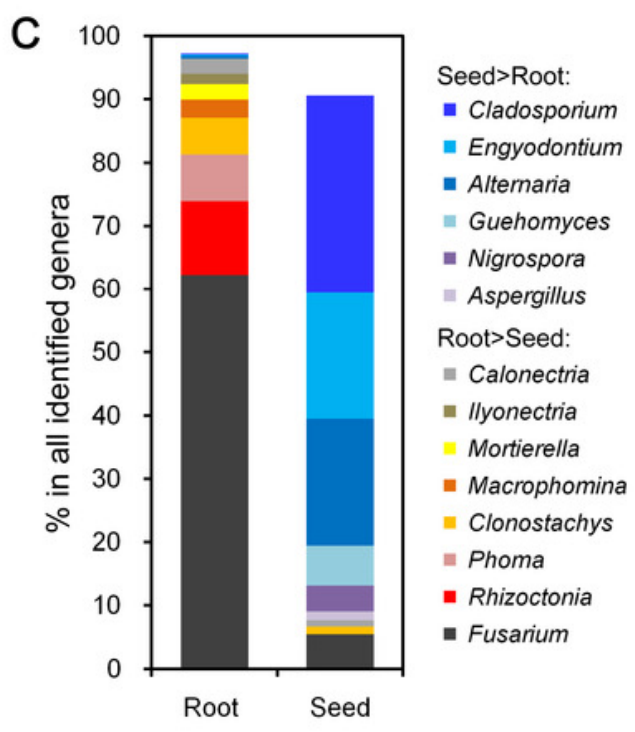

